\title{
Pharmacokinetics of Catechols in Human Subjects Intravenously Receiving XueBiJing Injection, an Emerging Antiseptic Herbal Medicine
}

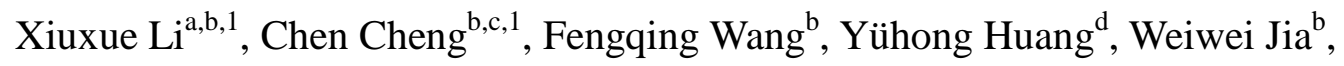
Olajide E. Olaleye ${ }^{\mathrm{b}}$, Meijuan $\mathrm{Li}^{\mathrm{b}}$, Yanfen $\mathrm{Li}^{\mathrm{d}}$, Chuan $\mathrm{Li}^{\mathrm{a}, \mathrm{b}, \mathrm{c}, *}$

${ }^{\mathrm{a}}$ Graduate School, Tianjin University of Traditional Chinese Medicine, Tianjin 300193, China;

${ }^{\mathrm{b}}$ Laboratory for DMPK Research of Herbal Medicines, Shanghai Institute of Materia Medica, Chinese Academy of Sciences, Shanghai 201203, China;

${ }^{\mathrm{c}}$ Institute of Chinese Materia Medica, China Academy of Chinese Medical Sciences, Beijing 100700, China;

${ }^{\mathrm{d}}$ Second Affiliated Hospital, Tianjin University of Traditional Chinese Medicine, Tianjin 300150, China;

Running title: Pharmacokinetics of XueBiJing Catechols in Human Subjects

* Corresponding author: Chuan Li, Ph.D., Laboratory for DMPK Research of Herbal Medicines, Shanghai Institute of Materia Medica, Chinese Academy of Sciences, 501 Haike Road, Zhangjiang Hi-Tech Park, Shanghai 201203, China. Tel. +86-21-50803106, Fax.+86-21-50803106, E-mail: chli@ simm.ac.cn (C. Li)

${ }^{1}$ These authors contributed equally to this work. 


\section{A B S T R A C T}

XueBiJing injection, prepared from a five-herb combination, is extensively used as add-on therapy in routine sepsis care in China. Catechols, derived from the component herb Salvia miltiorrhiza roots (Danshen), are probably important because of their reported antiseptic properties. This study was designed to characterize pharmacokinetics of major circulating Danshen catechols in human subjects intravenously receiving the injection at the label doses. A total of 17 Danshen catechols were detected in XueBiJing injection (content level, 0.1-139.3 $\mu \mathrm{M}$ ). After dosing, tanshinol and salvianolic acid B exhibited relatively high levels of systemic exposure with mean elimination half-lives of 0.38 and 0.29 hour, respectively. The total plasma clearance and apparent volume of distribution at steady state of tanshinol were $1.07 \mathrm{~L} / \mathrm{h} / \mathrm{kg}$ and $0.40 \mathrm{~L} / \mathrm{kg}$, respectively, whereas those of salvianolic acid B were $0.43 \mathrm{~L} / \mathrm{h} / \mathrm{kg}$ and $0.13 \mathrm{~L} / \mathrm{kg}$, respectively. Protocatechuic acid and five other catechols were also detected in plasma but at low exposure levels. Although protocatechuic aldehyde had the highest content level in the injection, like the remaining eight catechols, it was undetected in plasma. Protocatechuic aldehyde was extensively converted into protocatechuic acid and other metabolites. The information gained here facilitates understanding the roles of Danshen catechols in therapeutic actions of XueBiJing injection.

\section{Keywords:}

XueBiJing injection

Salvia miltiorrhiza roots

Danshen

Catechol

Pharmacokinetics

Sepsis 


\section{Introduction}

Sepsis, an infection-induced systemic inflammatory response, is one of the most pressing clinical problems with significant mortality rate and poor prognosis [1]. In China, herbal medicines are often used as add-on therapy in the routine sepsis care [2]. One of such herbal medicines is XueBiJing injection, which was approved by the China Food and Drug Administration (China FDA; Beijing, China), in 2004, for treatment of sepsis and multiple organ dysfunction syndromes. The injection is prepared from a five-herb combination of Carthamus tinctorius flowers (Honghua in Chinese/Benibana in Japanese), Paeonia lactiflora roots (Chishao/Aka-shakuyaku), Ligusticum chuanxiong rhizomes (Chuanxiong/Senkyu), Angelica sinensis roots (Danggui/Toki), and Salvia miltiorrhiza roots (Danshen/Tanjin) and is a sterile and nonpyrogenic formulation for intravenous administration. Clinical studies show that treatment with XueBiJing injection reduces 28-day mortality and incidence of complications, improves acute physiology and chronic health evaluation II score and prognosis, and shortens stay in intensive care unit and ventilation time in septic patients with low incidence of side effects $[3,4]$. The herbal therapy improves microcirculation, alleviates coagulation and inflammation, protects the endothelium, and regulates immune response [5,6].

Four classes of compounds (including Honghua flavonoids, Chishao monoterpene glycosides, Chuanxiong/Danggui phthalides, and Danshen catechols) are probably important for XueBiJing injection, because they have been reported to have antiseptic and related properties. However, little is known about which of these herbal compounds are responsible for the injection's therapeutic actions. This is determined not only by the compounds' pharmacological properties and potency but also by their reachability for and concentrations at the sites of action. Accordingly, 
pharmacokinetic research is required, which is based on the four classes of XueBiJing compounds. This study was designed to assess systemic exposure to Danshen catechols in human subjects intravenously receiving XueBiJing injection and to characterize the pharmacokinetics of major circulating compounds.

\section{Materials and Methods}

A detailed description of materials and methods is provided in the Appendix A. Supplementary information, which is available online.

\subsection{XueBiJing injection and Danshen}

Samples of eight lots of XueBiJing injection (China FDA ratification number, GuoYaoZhunZi-Z20040033) and raw material samples of Danshen (S. miltiorrhiza roots) were obtained from Tianjin Chasesun Pharmaceutical Co., Ltd. (Tianjin, China).

\subsection{Chemicals and reagents}

Reference standards of Danshen catechols (purity, $\geq 98 \%$ by HPLC) were obtained from Tauto Biotech (Shanghai, China). 3-O-Methyltanshinol and 4-O-methyltanshinol ( $\geq 98 \%$ by HPLC) were synthesized in-house using the methods by Tian et al. [7]

\subsection{Human study}

An ethics committee of clinical investigation at the Second Affiliated Hospital of Tianjin University of Traditional Chinese Medicine (Tianjin, China) reviewed and approved the protocol for human pharmacokinetic study of XueBiJing injection. Healthy volunteers (19-31 years) were recruited and gave their informed consent to participate in the study, which was implemented at a national clinical research center 
of the Hospital (Tianjin, China). The human subjects were randomly divided into three groups (six male and six female subjects for each). The test dosage regimens of XueBiJing injection were set based on the injection's label dose $(50 \mathrm{~mL} /$ person for treatment of sepsis or $100 \mathrm{~mL} /$ person for treatment of multiple organ dysfunction syndromes) as used in clinics; they were: a 75-minute intravenous infusion of 100-mL XueBiJing injection diluted in $100-\mathrm{mL} \mathrm{NaCl}$ injection (group I), a 150-minute intravenous infusion of $100-\mathrm{mL}$ XueBiJing injection diluted in $200-\mathrm{mL} \mathrm{NaCl}$ injection (group II), and a 75-minute intravenous infusion of 50-mL XueBiJing injection diluted in 100-mL NaCl injection (group III). Each subject received one of the three regimens. After receiving the single dose of XueBiJing injection, the six male subjects of group III continued to receive the same dose of the injection each day for the following six days. Blood and urine were sampled.

\subsection{Liquid Chromatography/Mass Spectrometry-Based Assays}

An AB Sciex API 4000 Q Trap mass spectrometer (Toronto, Canada), interfaced via a Turbo V ion source with a Waters Acquity UPLC separation module, was used for detection and quantification of Danshen catechols and metabolites.

\subsection{Data Processing}

Pharmacokinetic parameters were estimated using non-compartmental analysis with Kinetica software (version 5.0; Thermo Scientific, Philadelphia, PA, USA) and are shown as means \pm standard deviation. Statistical analysis was performed using IBM SPSS Statistics software (version 19.0; Somers, NY, USA). A $P$ value $<0.05$ was considered statistically significant.

\section{Results and Discussion}

Cell- and animal-based studies showed that Danshen catechols have 
antithrombotic, endothelial protective, vasodilating, cardioprotective, anti-inflammatory, and antioxidant properties [8], which could be important for XueBiJing therapy. A total of 17 Danshen catechols were detected in XueBiJing injection (Appendix B. Supplementary data). Different from raw materials of Danshen exhibiting predominant salvianolic acid B (15), XueBiJing injection contained higher content levels of protocatechuic aldehyde (1) and tanshinol (3) than 15. Salvianolic acid $\mathrm{B}$ was reported to be converted into protocatechuic aldehyde, tanshinol and other catechols by heating in water [9]. This conversion probably occurred during the injection's preparation. The injection exhibited lot-to-lot variability in content levels of $\mathbf{1}, \mathbf{3}$, rosmarinic acid (5), isosalvianolic acid C (9), 15, and protocatechuic acid (2) with relative standard deviations of $12.7-23.4 \%$. Such values for the remaining minor catechols were $0.0-36.4 \%$. The XueBiJing injection with a lot number of 1309301 was used in human PK study. The dose levels of catechols from this XueBiJing injection (100 mL/person) could be divided into three classes: $>10 \mu \mathrm{mol} /$ person (1), $1-10 \mu \mathrm{mol} /$ person $(\mathbf{3}, \mathbf{5}, \mathbf{9}, \mathbf{1 5}$, and $\mathbf{2})$, and $<1 \mu \mathrm{mol} /$ person (the remaining minor catechols). The total dose level of the minor catechols was only $4.0 \%$ of the total dose of catechols present in the injection.

Eight unchanged Danshen catechols were detected in plasma during and after a single 75-minute intravenous infusion of $100 \mathrm{~mL}$ XueBiJing injection (diluted in 100 $\mathrm{mL}$ of $\mathrm{NaCl}$ injection) per subject (Fig. 1A); these compounds were undetected in plasma before dosing. The detected catechols were tanshinol (3), rosmarinic acid (5), isosalvianolic acid C (9), salvianolic acid B (15), protocatechuic acid (2), salvianolic acid C (10), lithospermic acid (13), and salvianolic acid D (8); they were also detected in urine (Fig. 1B). Unchanged protocatechuic aldehyde (1) was not detected in plasma; it was extensively converted into $\mathbf{2}$, which was further methylated and glucuronized to 
form $\mathbf{M} \mathbf{2}_{\mathbf{M - G}-\mathbf{1}}$ and $\mathbf{M} \mathbf{2}_{\mathbf{M - G - 2}}$. Two methylated metabolites of $\mathbf{3}\left(\mathbf{M} \mathbf{3}_{\mathbf{M - 1}}\right.$ and $\left.\mathbf{M} \mathbf{3}_{\mathbf{M - 2}}\right)$ and one dimethylated metabolite of $\mathbf{5}\left(\mathbf{M 5}_{\mathbf{2 M}}\right)$ were also detected in plasma (Fig. 1C). By comparing with the synthesized compounds, $\mathbf{M} \mathbf{3}_{\mathbf{M - 1}}$ and $\mathbf{M} \mathbf{3}_{\mathbf{M}-2}$ were characterized as 3-O-methyltanshinol and 4-O-methyltanshinol, respectively. The preceding catechol metabolites were also detected in urine (Fig. 1D).

Figure 1 (E-G) shows plasma concentrations of tanshinol (3), salvianolic acid B (15), and protocatechuic acid (2) over time during and after an intravenous infusion of XueBiJing injection. Table $\mathbf{1}$ summarizes the pharmacokinetic parameters of $\mathbf{3}$ and $\mathbf{1 5 .}$ The plasma $C_{\max }$ values of $\mathbf{3}$ and $\mathbf{1 5}$ were measured just before stopping the infusion. After the infusion was stopped, their plasma concentration declined with mean $t_{1 / 2}$ values of $0.38 \pm 0.14$ and $0.29 \pm 0.08$ hour, respectively. The gender differences were insignificant for $\mathbf{3}$ and $\mathbf{1 5}$ with respect to $C_{\max }$ and $\mathrm{AUC}_{0-\infty}$, when the doses were corrected for body weight $(P>0.05)$. Although the levels of systemic exposure were XueBiJing-dose-dependent $(P=0.0000005-0.005)$, the dosage regimens had no significant effect on the $V_{\mathrm{SS}}$ and $\mathrm{CL}_{\mathrm{tot}, \mathrm{p}}$ of $\mathbf{3}(P>0.05)$ or on those of $\mathbf{1 5}(P>0.05)$. The mean $V_{\mathrm{SS}}$ of $\mathbf{3}$ for all the groups $(0.40 \pm 0.19 \mathrm{~L} / \mathrm{kg})$ was only slightly larger than the human total extracellular volume $(0.26 \mathrm{~L} / \mathrm{kg})$, whereas that of $15(0.13 \pm 0.04 \mathrm{~L} / \mathrm{kg})$ was smaller. This suggested that both catechols had small volume of distribution. The mean $\mathrm{CL}_{\text {tot,p }}$ of $\mathbf{3}$ for all the groups $(1.07 \pm 0.21 \mathrm{~L} / \mathrm{hour} / \mathrm{kg})$ and that of $\mathbf{1 5}(0.43 \pm 0.08$ L/hour $/ \mathrm{kg}$ ) were $44 \%$ and $18 \%$ of human cardiac plasma output $(2.4 \mathrm{~L} / \mathrm{hour} / \mathrm{kg})$, respectively, suggesting the two catechols had high and moderate total clearance, respectively. Neither 3 nor 15 exhibited any gender differences in $t_{1 / 2}, V_{\mathrm{SS}}$, or $\mathrm{CL}_{\mathrm{tot}, \mathrm{p}}$ $(P>0.05)$. Table 1 shows the mean $C_{\mathrm{SS}}$ and $A_{\mathrm{SS}}$ values of $\mathbf{3}$ and those of $\mathbf{1 5}$ for test dosage regimens of XueBiJing injection. No significant accumulation was observed for plasma 3 and $\mathbf{1 5}$ in $\mathrm{AUC}_{0-\infty}$ during multiple infusions of XueBiJing injection 
$(P>0.05)$ and the $R_{\mathrm{ac}}$ values were 1.04 and 0.96 , respectively. Elimination of $\mathbf{3}$ was mainly via renal excretion with $\mathrm{CL}_{\mathrm{R}} / \mathrm{CL}_{\text {tot,p }}$ ratios of $63.0-73.8 \%$. Renal excretion of tanshinol was found to be attributed mainly to OAT1/2/3-mediated tubular secretion [11]. Renal excretion of $\mathbf{1 5}$ was poor. Salvianolic acid B was reported to be eliminated, in rats, mainly via biliary excretion of its methylated metabolites [12].

Herein, we identified Danshen compounds, which exhibited relatively high levels of systemic exposure after dosing XueBiJing injection, and assessed their pharmacokinetics. This enabled us to compare the pharmacokinetic concentrations of Danshen catechols with the reported effective concentrations for exerting their antiseptic activities. The results suggested that the concentrations of Danshen catechols measured in human plasma after dosing XueBiJing injection might be too low, when compared with the reported effective concentrations [8]. Although the low concentrations do not result in ineffectiveness of XueBiJing injection in treatment of sepsis, they probably limit the contribution of Danshen to the current XueBiJing therapy. The differences in Danshen catechols' concentrations in human plasma between dosed XueBiJing injection and dosed DanHong injection [10] suggested that higher plasma concentrations are technically achievable for future XueBiJing therapy. This idea is important for design and development of new and superior antiseptic agents derived from the current XueBiJing injection. 


\section{Acknowledgements}

This work was funded by grants from the National Science \& Technology Major Project of China 'Key New Drug Creation and Manufacturing Program' (2009ZX09304-002), the National Science Foundation of China for Distinguished Young Scholars (30925044), National Natural Science Foundation of China (81503345), and China Postdoctoral Science Foundation funded project (2014M560169). 


\section{References}

[1] Angus DC, van der Poll T. Severe sepsis and septic shock. N Engl J Med 2013;369: 840-51.

[2] Chinese Society of Critical Care Medicine: Chinese guidelines for management of severe sepsis and septic shock 2014. Chin Crit Care Med 2015;27: 401-26.

[3] Chen Y-X, Li C-S. The effectiveness of XueBiJing injection in therapy of sepsis: a multicenter clinical study. Chin J Emerg Med 2013;22:130-35.

[4] Gao J, Kong L-B, Liu S, Feng Z-Q, Shen H, Liu Q-Q. A prospective multicenter clinical study of XueBiJing injection in the treatment of sepsis and multiple organ dysfunction syndrome. Chin Crit Care Med 2015;27:465-70.

[5] Yin Q, Li C-S. Treatment effects of XueBiJing injection in severe septic patients with disseminated intravascular coagulation. Evidence-Based Complement Alternat Med 2014;2014:949254.

[6] He J-Z, Tan Z-P, Zhang M-Z, Guo L-H. Effect of XueBiJing injection on hemodynamics and endothelial function in patients with severe sepsis: a prospective study. Chin Crit Care Med 2015;27:127-32.

[7] Tian D-D, Jia W-W, Liu X-W, Wang D-D, Liu J-H, Dong J-J, et al. Methylation and its role in the disposition of tanshinol, a cardiovascular carboxylic catechol from Salvia miltiorrhiza roots (Danshen). Acta Pharmacol Sin 2015;36:627-43.

[8] Jiang R-W, Lau K-M, Hon P-M, Mak T-C, Woo K-S, Fung K-P. Chemistry and biological activities of caffeic acid derivatives from Salvia miltiorrhiza. Curr Med Chem 2005;12:237-46.

[9] Guo Y-X, Zhang D-J, Wang H, Xiu Z-L, Wang L-X, Xiao H-B. Hydrolytic kinetics of lithospermic acid B extracted from roots of Salvia miltiorrhiza. J Pharm Biomed Anal 2007;43:435-39.

[10] Li M-J, Wang F-Q, Huang Y-H, Du F-F, Zhong C-C, Olaleye OE, et al. Systemic exposure to and disposition of catechols, derived from Salvia miltiorrhiza roots (Danshen), after intravenous dosing DanHong injection in human subjects, rats, and dogs. Drug Metab Dispos 2015;43:679-90.

[11] Jia W-W, Du F-F, Liu X-W, Jiang R-R, Xu F, Yang J-L, et al. Renal tubular secretion of tanshinol: molecular mechanisms, impact on its systemic exposure, and propensity for dose-related nephrotoxicity and for renal herb-drug interactions. Drug Metab Dispos 2015;43:669-78.

[12] Zhang Y, Akao T, Nakamura N, Hattori M, Yang X-W, Duan C-L, et al. Magnesium lithospermate B is excreted rapidly into rat bile mostly as methylated metabolites, which are potent antioxidants. Drug Metab Dispos 2004;32:752-57. 


\section{Figure Legends}

Fig. 1. Systemic exposure to, renal excretion of, and pharmacokinetics of Danshen catechols and metabolites in human subjects receiving an intravenous infusion of XueBiJing injection. For detection of unchanged and metabolized Danshen catechols (A-D), human subjects received a 75 -minute intravenous infusion of $100-\mathrm{mL}$ XueBiJing injection (diluted in $100-\mathrm{mL} \mathrm{NaCl}$ injection); the plasma samples of the same point of time $(n=12)$ were pooled for analysis, whereas the urine samples of the same period of time $(n=12)$ were also pooled. Red and black bars are used to show the plasma and urinary levels of the catechols, respectively. The detected plasma (A) and urine Danshen catechols (B) are ranked according to their content levels in XueBiJing injection and their chemical names are shown in Appendix B. Table S1. The symbol "×" (in red) denotes the compound that was not detected. Panels $\mathbf{E}-\mathbf{G}$ show the mean plasma concentrations of $\mathbf{3}$, salvianolic acid B (15), and protocatechuic acid (2) over time, respectively. Unlike the sample treatment for detection of catechols, the human plasma samples of the same point of time were not pooled before being analyzed for determination of the compounds' pharmacokinetics. Red lines: the subjects of group I receiving a 75-minute intravenous infusion of 100-mL XueBiJing injection (diluted in 100-mL NaCl injection); blue lines: the subjects of group II receiving a 150-minute intravenous infusion of 100-mL XueBiJing injection (diluted in 200-mL $\mathrm{NaCl}$ injection); and green lines: the subjects of group III receiving a 75-minute intravenous infusion of 50-mL XueBiJing injection (diluted in 100-mL NaCl injection). Solid and open circles are used to shown the plasma concentrations for male and female subjects, respectively. The plasma concentrations of $\mathbf{2}$ were quite low for Groups II and III, so the corresponding plasma concentration-time curves are not shown. 


\title{
Pharmacokinetics of Catechols in Human Subjects Intravenously Receiving XueBiJing Injection, an Emerging Antiseptic Herbal Medicine
}

Xiuxue Li, Chen Cheng, Fengqing Wang, Yühong Huang, Weiwei Jia, Olajide E. Olaleye, Meijuan Li, Yanfen Li, Chuan $\mathrm{Li}$

Drug Metabolism and Pharmacokinetics

\section{Appendix A. Supplementary Information}

\begin{abstract}
Materials and Methods
XueBiJing injection and Danshen: Samples of eight lots (1309271, 1309281, 1309291, 1309301, 1405301, 1406161, 1408191, and 1410081) of XueBiJing injection (China FDA ratification number, GuoYaoZhunZi-Z20040033) and raw material samples of Danshen (S. miltiorrhiza roots; lot number, 1206121, 1405181, and 1412071) were obtained from Tianjin Chasesun Pharmaceutical Co., Ltd. (Tianjin, China). The standard reference materials $S$. miltiorrhiza roots were obtained from the National Institutes for Food and Drug Control (Beijing, China).

Chemicals and reagents: Reference standards of tanshinol; protocatechuic aldehyde; protocatechuic acid; lithospermic acid; rosmarinic acid; and salvianolic acids A, B, C, and D (purity, $\geq 98 \%$ by HPLC) were obtained from Tauto Biotech (Shanghai, China). 3-O-Methyltanshinol and 4- $O$-methyltanshinol ( $\geq 98 \%$ by HPLC) were synthesized in-house using the methods by Tian et al. [1] A $0.9 \%$ sodium chloride injection ( $\mathrm{NaCl}$ injection; China FDA ratification number, GuoYaoZhunZi-H12020025) was manufactured by China Otsuka Pharmaceutical Co., Ltd. (Tianjin, China).
\end{abstract}

Human study: An ethics committee of clinical investigation at the Second Affiliated Hospital of Tianjin University of Traditional Chinese Medicine (Tianjin, China) reviewed and approved the protocol for human pharmacokinetic study of XueBiJing injection. In brief, 18 male and 18 female healthy volunteers (19-31 years) were recruited and gave their informed consent to participate in the study, which was implemented at a national clinical research center of the Hospital (Tianjin, China). The human subjects were randomly divided into three groups (six male and six female subjects for each), stratified by gender. The test dosage regimens of XueBiJing injection were set based on the injection's label dose $(50 \mathrm{~mL} /$ person for treatment of sepsis or $100 \mathrm{~mL} /$ person for treatment of multiple organ dysfunction syndromes) as used in clinics; they were: a 75-minute intravenous infusion of 100-mL XueBiJing injection (lot number, 1309301) diluted in 100-mL NaCl injection (group I), a 150-minute intravenous infusion of 100-mL XueBiJing injection (1309301) diluted in 200-mL NaCl injection (group II), and a 75-minute intravenous infusion of 50-mL XueBiJing injection (1309301) diluted in 100-mL NaCl injection (group III). Each subject received one of the three dosage regimens. Serial blood samples $(\sim 3 \mathrm{~mL})$ were collected from an antecubital vein catheter before and 0.17, 0.5, 1.25 (75 minutes), 1.42 (groups I and III only), 1.75 (groups I and III only), 2.25 (groups I and III only), 2.5 (150 minutes; group II only), 2.67 (group II only), 3 (group II only), 3.25 (groups I and III only), 3.5 (group II only), 4.5 (group II only), 5.25 (groups I and III only), 6.5 (group II only), 9.25 (groups I and III only), 10.5 (group II only), and 24 hours after starting the infusion and heparinized. Serial urine samples were also collected before and 0-3, 3-6, 6-10, and 10-24 hours after starting the infusion. After receiving the single dose of XueBiJing injection, the six male subjects of group III continued to receive the same dose of the herbal injection each day for the following six days. On days $2-6$, blood sampling was performed before and 1.25 hour after the daily infusion was started; on day 7, blood and urine samples were collected according to the time schedule same as that on day 1. The human study was registered in Chinese Clinical Trials Registry (www.chictr.org) with a registration number of ChiCTR-ONRC-13003932. 
Liquid Chromatography/Mass Spectrometry-Based Assays: An AB Sciex API 4000 Q Trap mass spectrometer (Toronto, Canada), interfaced via a Turbo V ion source with a Waters Acquity UPLC separation module, was used for detection and quantification of Danshen catechols and metabolites. Human plasma and urine samples $(50 \mu \mathrm{L})$ were acidified with $30 \mu \mathrm{L}$ formic acid, extracted with $500 \mu \mathrm{L}$ of ethyl acetate, and centrifuged; the supernatant were reduced to dryness and reconstituted with $50 \mu \mathrm{L}$ of $50 \%$ methanol before analysis. For detection of unchanged catechols and metabolites, chromatographic separation was achieved on a Waters Acquity UPLC HSS T3 1.8 - $\mu \mathrm{m}$ column $\left(100 \times 2.1 \mathrm{~mm}\right.$ i.d.; Dublin, Ireland; kept at $\left.40^{\circ} \mathrm{C}\right)$ using a mobile phase that consisted of solvent A (methanol/water, 1:99, v/v, containing $25 \mathrm{mmol} / \mathrm{L}$ formic acid) and solvent B (methanol/water, 99:1, v/v, containing $25 \mathrm{mmol} / \mathrm{L}$ formic acid). The mobile phase was delivered at $0.3 \mathrm{~mL} / \mathrm{min}$ and a gradient program was used as follows: 0-2 minutes, at $2 \%$ solvent $\mathrm{B} ; 2-32$ minutes, from $2 \%$ to $98 \%$ solvent $\mathrm{B} ; 32-37$ minutes, at $98 \%$ solvent $\mathrm{B}$; and $37-42$ minutes, at $2 \%$ solvent B. For the quantification, the chromatographic method was modified by using a shortened gradient program ( $0-3$ minutes, from $2 \%$ to $30 \%$ solvent $\mathrm{B} ; 3-5$ minutes, from $30 \%$ to $60 \%$ solvent $\mathrm{B}$; 5-5.1 minutes, from $60 \%$ to $2 \%$ solvent $\mathrm{B}$; and 5.1-6.5 minutes, at $2 \%$ solvent $\mathrm{B}$ ). The precursor-to-product ion pairs for multiple-reaction monitoring of plasma tanshinol, salvianolic acid B, and protocatechuic acid was achieved using the precursor-product ion pair $m / z, 197 \rightarrow 179,717 \rightarrow 519$, and $153 \rightarrow 109$, respectively. Matrix-matched calibration curves $(4.6-1111 \mathrm{nM})$ were constructed using weighted $\left(1 / X^{2}\right)$ linear regression of the analyte area $(Y)$ against the corresponding nominal analyte concentration $(X, \mathrm{nmol} / \mathrm{L})$. Before use, the quantification assay was validated according to the US Food and Drug Administration guide on bioanalytical method validation (2013). The lower limits of quantification were 4.6, 4.6, and $13.7 \mathrm{nmol} / \mathrm{L}$ for measurement of tanshinol, salvianolic acid $\mathrm{B}$, and protocatechuic acid in plasma samples, respectively (the assay precision, $<20 \%$; the assay accuracy, $80-120 \%$ ).

Data Processing: Pharmacokinetic parameters were estimated using non-compartmental analysis with Kinetica software (version 5.0; Thermo Scientific, Philadelphia, PA, USA) and are shown as means \pm standard deviation. Statistical analysis was performed using IBM SPSS Statistics software (version 19.0; Somers, NY, USA). A P value $<0.05$ was considered statistically significant.

\section{References}

1) Tian D-D, Jia W-W, Liu X-W, Wang D-D, Liu J-H, Dong J-J, et al. Methylation and its role in the disposition of tanshinol, a cardiovascular carboxylic catechol from Salvia miltiorrhiza roots (Danshen). Acta Pharmacol Sin 2015;36:627-43.

\section{Brief Description of Method for Preparation of XueBiJing Injection}

XueBiJing injection was a 1:2 injectable solution, two milliliters of which was prepared from a combination of $0.2 \mathrm{~g}$ each of Carthamus tinctorius flowers (Honghua), Paeonia lactiflora roots (Chishao), Ligusticum chuanxiong rhizomes (Chuanxiong), Angelica sinensis roots (Danggui), and Salvia miltiorrhiza roots (Danshen). Honghua was first extracted with $30 \%$ ethanol and then the extract was diluted with ethanol and centrifuged. The supernatant was reduced followed by dilution with water. After centrifugation, the supernatant was reduced and precipitated with ethanol; after centrifugation again, the supernatant was reduced to yield "H-extract". Chishao was first extracted with water. The aqueous extract was concentrated under reduced pressure, precipitated with ethanol, and filtered. The filtrate was then reduced and extracted with water-saturated n-butanol. After the n-butanol extract was reduced to dryness, the residual was dissolved in water to yield "C-extract". Chuanxiong, Danggui, and Danshen were combined and extracted with water. The aqueous extract was treated, in the same way as the preparation of "C-extract", to yield "CDD-extract". The preceding H-extract, C-extract, and CDD-extract were combined, diluted with isotonic glucose solution, and fortified with Tween 80. The solution was adjusted to $\mathrm{pH}$ 5.8-6.2, ultrafiltered, and sterilized. The finished product of XueBiJing injection was standardized to contain $1.0-1.7 \mathrm{mg} / \mathrm{mL}$ concentration of paeoniflorin, $0.2-0.5 \mathrm{mg} / \mathrm{mL}$ concentration of hydroxysafflor yellow A, and $0.01-0.20 \mathrm{mg} / \mathrm{mL}$ concentration of benzoic acid. 

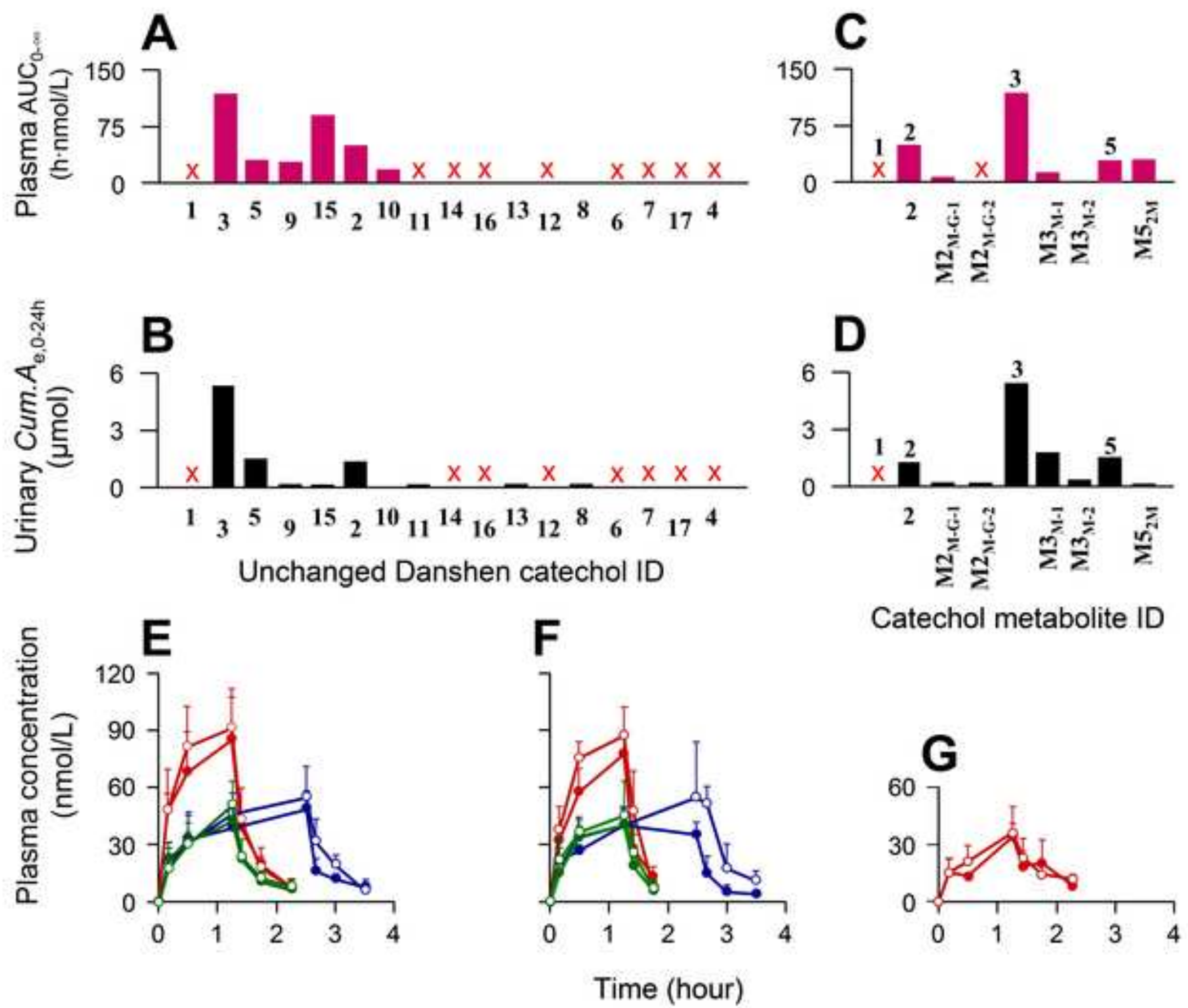

Catechol metabolite ID

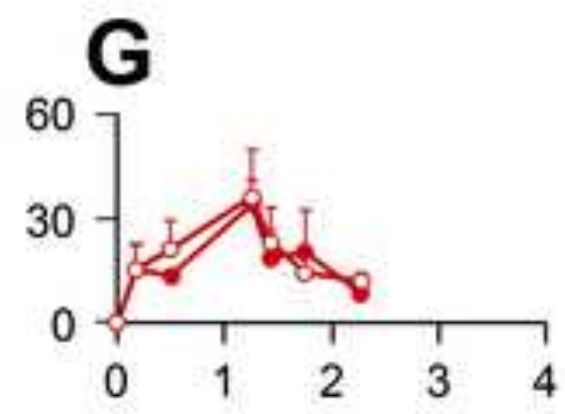

Time (hour) 
Table 1

Pharmacokinetics of Danshen catechols and metabolites in human subjects intravenously receiving XueBiJing injection.

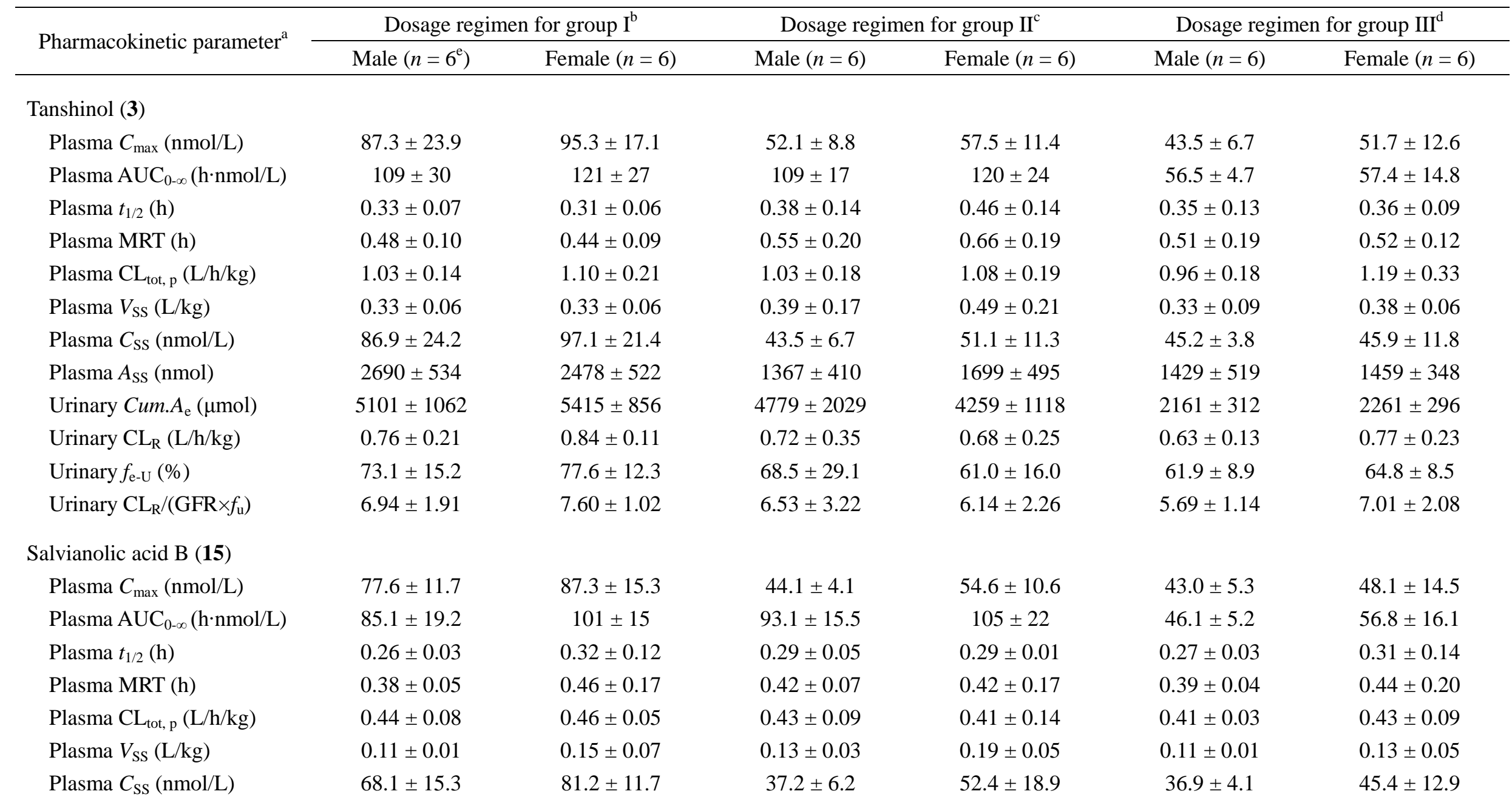




\begin{tabular}{|c|c|c|c|c|c|c|}
\hline Plasma $A_{\mathrm{SS}}(\mathrm{nmol})$ & $779 \pm 116$ & $926 \pm 345$ & $427 \pm 67$ & $425 \pm 174$ & $393 \pm 38$ & $446 \pm 197$ \\
\hline Urinary $C u m . A_{\mathrm{e}}(\mu \mathrm{mol})$ & $5.18 \pm 1.58$ & $5.61 \pm 2.27$ & $5.14 \pm 2.35$ & $6.60 \pm 3.22$ & - & - \\
\hline Urinary $\mathrm{CL}_{\mathrm{R}}(\mathrm{L} / \mathrm{h} / \mathrm{kg})$ & $0.001 \pm 0.000$ & $0.001 \pm 0.000$ & $0.001 \pm 0.000$ & $0.001 \pm 0.000$ & - & - \\
\hline Urinary $f_{\mathrm{e}-\mathrm{U}}(\%)$ & $0.21 \pm 0.06$ & $0.22 \pm 0.09$ & $0.26 \pm 0.03$ & $0.22 \pm 0.13$ & - & - \\
\hline Urinary $\mathrm{CL}_{\mathrm{R}} /\left(\mathrm{GFR} \times f_{\mathrm{u}}\right)$ & $0.008 \pm 0.002$ & $0.007 \pm 0.001$ & $0.009 \pm 0.003$ & $0.007 \pm 0.002$ & - & - \\
\hline
\end{tabular}

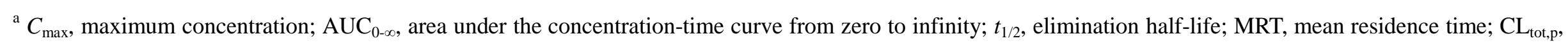
total plasma clearance; $V_{\mathrm{SS}}$, apparent volume of distribution at steady state; $C_{\mathrm{SS}}$, calculated concentration at steady state; $A_{\mathrm{SS}}$, calculated amount at steady state; Cum. $A_{\mathrm{e}}$, cumulative amount excreted into urine; $\mathrm{CL}_{\mathrm{R}}$, renal clearance; $f_{\mathrm{e}}$, fraction of dose excreted into urine; GFR, glomerular filtration rate.

${ }^{\mathrm{b}}$ Dosage regimen for group I: a 75 -minute intravenous infusion of $100-\mathrm{mL}$ XueBiJing injection (diluted in $100-\mathrm{mL} \mathrm{NaCl}$ injection).

${ }^{\mathrm{c}}$ Dosage regimen for group II: a 150-minute intravenous infusion of 100-mL XueBiJing injection (diluted in 200-mL NaCl injection).

${ }^{\mathrm{d}}$ Dosage regimen for group III: a 75-minute intravenous infusion of 50-mL XueBiJing injection (diluted in 100-mL NaCl injection).

e The human plasma samples of the same point of time were not pooled before being analyzed for determination of the compounds' pharmacokinetics, whereas the human urine samples of the same period of time were also not pooled. 